\title{
On the dilatational wave motion in anisotropic fractal solids
}

\author{
Hady Joumaa, Martin Ostoja-Starzewski \\ Department of Mechanical Science and Engineering, University of Illinois at \\ Urbana-Champaign, 1206 W. Green St., Urbana, IL 61801, USA
}

\begin{abstract}
This paper reports a study of wave motion in a generally anisotropic fractal medium (i.e. with different fractal dimensions in different directions), whose constitutive response is represented by an isotropic Hooke's law. First, the governing elastodynamic laws are formulated on the basis of dimensional regularization. It is discovered that the satisfaction of the angular momentum equation precludes the implementation of the classical elasticity theory which results in symmetry of the Cauchy stress tensor. Nevertheless, the classical elastic constitutive model can still be applied to explore dilatational wave propagation; in such a case, the angular momentum balance is "trivially" satisfied. The resulting problem, of eigenvalue type, is solved analytically. A computational finite element method solver is also developed to simulate the problem in its $1 \mathrm{~d}$ form, it is validated by the reference solutions generated through the modal analysis. A 3d finite-difference-based solver is also developed and the obtained results match those of the $1 \mathrm{~d}$ simulation.

Keywords: elastodynamics, fractal solid, dilatational wave
\end{abstract}




\section{Introduction}

Fractal mechanics is a current area of research in science and engineering. Fractal media are abundant in nature (rocks, tree leaves) and in living bodies (brain). However, the conventional and well-known continuum mechanics lacks a modelling capability for such materials, and that is the issue motivating the growing activity in the so-called fractal mechanics [5]. Materials which exhibit a highly irregular (non-smooth) topology or inherent porosity are often treated in the fractal framework where a dimensional regularization (homogenization technique) is applied to map their fractal geometry to an equivalent Euclidean one. In general, fractal materials are of two types: random (natural) or regular (geometric); the latter exhibits a perfectly defined fractality, i.e. its Hausdorff dimension is mathematically determined via the self-similarity property applied in its construction [9], while the former has a statistical fractality. The development of a field theory in which the fundamental laws of mechanics (conservation of mass, linear and angular momentum) are reproduced in the fractal setting resulting in continuum-type equations is the current focus of many scientists $[18,14]$.

Some fractal media can be classified as isotropic, but in general, fractals are anisotropic, i.e. their Hausdorff dimension is direction-dependent. Wave propagation in isotropic fractal media on spherical domains has been studied in $[11,18]$, whereas a formulation of a three-dimensional (3d) elastodynamic model for anisotropic fractal solids was developed in [14] based on product measures. The latter work utilized two different approaches to derive the governing elastodynamic equations of this material model: the fractal mechanics approach and the variational energy approach with both 
approaches producing consistent results. This attribute is not realized in previous fractal analysis research where a single approach is followed to describe the mechanics of the model. In brief, Li and Ostoja-Starzewski [14] adopted the modified Riemann-Liouville fractional integral formulated by Jumarie [13] to derive the mass power law, Gauss's law and the transport theorems in fractal domains. Accordingly, the fractional integral of a certain function $f(x)$ over a fractal set of Hausdorff dimension $D \in] 0,1]$, with support on $[0, L]$, is regularized as follows

$$
\int_{0}^{L} f(x)(\mathrm{d} x)^{D}=\int_{0}^{L} D(L-x)^{D-1} f(x) \mathrm{d} x
$$

In this regard, the mass power law of a $3 \mathrm{~d}$ fractal body $\left(V_{D}\right)$ embedded in the Cartesian system $\mathrm{Ox}_{1} x_{2} x_{3}$ is derived as follows

$$
\begin{aligned}
m & =\int_{V_{D}} \rho\left(x_{i}\right) \mathrm{d} V_{D} \\
& =\int_{0}^{L_{1}} \int_{0}^{L_{2}} \int_{0}^{L_{3}} \rho\left(x_{1}, x_{2}, x_{3}\right)\left(\mathrm{d} x_{3}\right)^{D_{3}}\left(\mathrm{~d} x_{2}\right)^{D_{2}}\left(\mathrm{~d} x_{1}\right)^{D_{1}} \\
& =\int_{0}^{L_{1}} \int_{0}^{L_{2}} \int_{0}^{L_{3}} \rho\left(x_{1}, x_{2}, x_{3}\right) c_{1} c_{2} c_{3} \mathrm{~d} x_{3} \mathrm{~d} x_{2} \mathrm{~d} x_{1} \\
& =\int_{V} \rho\left(x_{i}\right) c_{V} \mathrm{~d} V
\end{aligned}
$$

where $\rho$ is the local mass density of the body and $c_{i}$ is the product measure along the $i$ th direction defined as

$$
c_{i}=D_{i}\left(L_{i}-x_{i}\right)^{D_{i}-1} \quad i=1,2,3
$$

and $c_{V}$ is a volumetric product measure defined as the product of all three product measures, thus $c_{V}=c_{1} c_{2} c_{3}$. Note that for a uniform density $\rho \equiv \rho_{0}$, the total mass becomes $m=\rho_{0} L_{1}^{D_{1}} L_{2}^{D_{2}} L_{3}^{D_{3}}$. This finding is mathematically 
consistent with the universal mass power law of a fractal body with characteristic length $L$ and Hausdorff dimension $D, m \sim L^{D}$ [18]. In conclusion, $c_{i}$ is applied to regularize $1 \mathrm{~d}$ or length integrals, while its equivalent, $c_{V}$, regularizes $3 \mathrm{~d}$ or volume integrals. Consistent with this, a surface product measure is introduced to regularize surface integrals as will be later shown in deriving the Green-Gauss theorem on fractal media.

At this point, we discuss the fractional reproduction of the Green-Gauss theorem, a widely applied integral identity in formulating many continuum mechanics principles. This analysis will result in defining the mathematical form of the fractal derivative, which will be utilized in generalizing the fundamental balance laws for mechanics in fractal media. In continuous domains, the Green-Gauss theorem correlates the integral of a vector function $f_{i}$ over a closed surface $\partial W$, to the integral of its divergence over the volume $W$ enclosed by this surface [17], thus

$$
\int_{\partial W} f_{i} n_{i} \mathrm{~d} S=\int_{W} f_{i, i} \mathrm{~d} V
$$

For fractal domains, the surface integral is regularized by the surface product measure in similarity with the regularization performed along the length as shown in Eq. (1). Thus, after considering the surface projection onto each coordinate plane as illustrated in [14], the surface integral becomes

$$
\int_{\partial W_{D}} f_{i} n_{i} \mathrm{~d} S_{D}=\int_{\partial W} f_{i} c_{i}^{S} n_{i} \mathrm{~d} S
$$

where $c_{i}^{S}=\frac{c_{V}}{c_{i}}$. Note that $c_{i}^{S}$ is independent of the $i$ th Cartesian coordinate $x_{i}$. Applying the conventional Green-Gauss theorem to the right-hand side of Eq. (5), we obtain

$$
\int_{\partial W} f_{i} c_{i}^{S} n_{i} \mathrm{~d} S=\int_{W}\left(f_{i} c_{i}^{S}\right)_{, i} \mathrm{~d} V=\int_{W} f_{i, i} c_{i}^{S} \mathrm{~d} V
$$


and using the volume integral regularization as was done in Eq. (2), the volume integral of Eq. (6) is expressed in the fractal configuration as

$$
\int_{W} f_{i, i} c_{i}^{S} \mathrm{~d} V=\int_{W_{D}} f_{i, i} \frac{c_{i}^{S}}{c_{V}} \mathrm{~d} V_{D}=\int_{W_{D}} \frac{1}{c_{i}} f_{i, i} \mathrm{~d} V_{D}
$$

Combining the integral identities of equations (5), (6), and (7), the generalization of the Green-Gauss theorem on fractal domains is reproduced. Thus

$$
\int_{\partial W_{D}} f_{i} n_{i} \mathrm{~d} S_{D}=\int_{W_{D}} \frac{1}{c_{i}} f_{i, i} \mathrm{~d} V_{D}
$$

By comparing equations (4) and (8), the mathematical form of the regularized fractal derivative can be clearly inferred. This fractal derivative, denoted by $\nabla_{i}^{D}$, is defined as

$$
\nabla_{i}^{D}=\frac{1}{c_{i}} \frac{\partial}{\partial x_{i}} \quad(\text { no sum on } i)
$$

As a first insight, this fractal derivative differs from the conventional fractional one by having the derivative of a constant to be zero. In fact, this derivative is applied to develop the fundamental balance laws of mechanics for a fractal medium with finite spatial extension, i.e., its fractal mass is distributed within a limited (internal) space, generally, in a non-homogeneous pattern. In our case, we consider a generalized anisotropic fractal body defined in the $3 \mathrm{~d}$ Cartesian system and having $L_{i}$ to be the size along the $i$ th direction. Thus the mechanics is valid only for $0<x_{i}<L_{i}$, and any attempt to analyse the mechanics outside this range is, in principle unphysical, and leads to mathematical fallacies.

Fractal media involved in real life applications can be described as "prefractals", which connotes a physical object exhibiting fractal features within a length scale of lower and upper cutoffs. The upper cutoff is indeed $L$ 
that appears in the fractal derivative operator, and the lower cutoff is the size of the smallest fractal feature within the object. The development of a continuum-like model for pre-fractals is discussed in detail in [14] where the governing equations of elastodynamics are formulated. In our paper, we briefly present the basic equations relevant to the dilatational wave propagation problem we intend to explore. The resulting eigenvalue type problem, is treated analytically and numerically in three different settings of boundary conditions.

\section{Elastodynamic model of dilatational wave motion}

The main objective of our work is the understanding of the propagation of dilatational waves in anisotropic fractal solids through analytical and computational means. In this section, we introduce the fractal version of the balance laws, then, we construct the appropriate elastodynamic model which suits the simulation of this particular type of waves. We also present the $3 \mathrm{~d}$ anisotropic fractal domain (Carpinteri column) on which the problem is solved.

With regard to kinematics, the strain tensor field for this fractal solid model resulting from an infinitesimal deformation $u_{i}$ is defined as

$$
\epsilon_{i j}=\frac{1}{2}\left(\nabla_{j}^{D} u_{i}+\nabla_{i}^{D} u_{j}\right)
$$

Similarly to the classical continuum case, the symmetry property of the strain tensor is preserved. The linear momentum balance incorporating inertia, elastic and body forces is expressed as

$$
\rho \frac{\partial^{2} u_{i}}{\partial t^{2}}=\nabla_{j}^{D} \sigma_{i j}+f_{i}
$$


while the angular momentum balance is given as

$$
e_{i j k} \frac{\sigma_{j k}}{c_{j}}=0
$$

where $e_{i j k}$ is the Levi-Civita permutation tensor. In the continuum case $\left(D_{i}=1\right.$ and thus $\left.c_{i} \equiv 1\right)$, the angular momentum balance is automatically satisfied whenever the adopted constitutive law generates a symmetric Cauchy stress tensor. However, in the fractal case, the satisfaction of the angular momentum balance becomes hard to achieve because $c_{i} \neq c_{j}$ (the product measures depend on different coordinates and they can never be equivalent). Therefore, in mechanics of fractal solid bodies, the stress tensor cannot be symmetric, and as such, the well known constitutive law of Hooke's model defined as ( $\delta_{i j}$ being the Kronecker tensor),

$$
\sigma_{i j}=\lambda \epsilon_{k k} \delta_{i j}+2 \mu \epsilon_{i j}
$$

can no more be applied since it generates a symmetric stress tensor out of a symmetric strain tensor. Consequently, a fundamental attribute of the classical theory of elasticity is lost due to fractal effects, and this is remedied by the application of a non-classical elastic constitutive model (e.g. Cosserat model) to achieve a meaningful satisfaction of the balance laws for general problems [15]. Nevertheless, a careful observation of the above model reveals a possibility to implement Hooke's law while still satisfying the angular momentum balance. This satisfaction is achieved trivially by setting all shear components of the stress tensor to be identically zero i.e. $\sigma_{i j} \equiv 0$ for $i \neq j$. A feasible kinematic configuration that forces all the shear stress components and, thus, all the shear strain components to vanish exists and is unique; it 
is prescribed as

$$
u_{i} \equiv u_{i}\left(x_{i}\right)
$$

We thus have the displacement in a given direction dependent only on the coordinate of that direction. As a result, the propagated waves in this particular problem are of dilatational type. This type of waves is also designated as irrotational or primary [8]. It is worth noting that propagation of distortional or secondary waves cannot be studied within the framework of Hooke's model since this type of waves is based on shear effects that cannot be considered because of the symmetry problem discussed before.

The incorporation of Hooke's law into the linear momentum balance produces the fractal Navier equation. In the general anisotropic case, three different Hausdorff dimensions are present in this equation. The continuum model is reproduced whenever all three Hausdorff dimensions are set to one (and consequently all product measures become identically one). This is consistent with the initial assumptions about this fractal solid model. The indicial form of the fractal Navier equation is stated as

$$
\rho \ddot{u}_{i}=\frac{\lambda+\mu}{c_{V}}\left(\frac{c_{V} u_{j, i}}{c_{i} c_{j}}\right)_{, j}+\frac{\mu}{c_{V}}\left(\frac{c_{V} u_{i, j}}{c_{j} c_{j}}\right)_{, j}
$$

In contrary to the classical continuum case, Eq. (15) is only meaningful to study dilatational wave propagation problems. Enforcing the prescribed kinematics that simulates the propagation of this type of waves (Eq. (14)), we obtain a set of three $1 \mathrm{~d}$ decoupled equations that are similar in form, their expression in terms of (a) fractal and (b) conventional derivatives is

$$
\frac{\rho \ddot{u}_{i}}{\lambda+2 \mu}=\nabla_{i}^{D}\left(\nabla_{i}^{D} u_{i}\right)
$$




$$
\frac{\rho \ddot{u}_{i}}{\lambda+2 \mu}=\frac{u_{i, i i}}{c_{i}^{2}}-\frac{c_{i, i} u_{i, i}}{c_{i}^{3}}
$$

The above equation contains one index $i$ and no summation should be considered if index is repeated. The inherent relation between the dilatational wave propagation and model decoupling makes the solution to a $3 \mathrm{~d}$ problem feasible by solving three similar but independent problems of 1d type. This significantly facilitates the computational analysis with regard to problem cost and complexity.

In the remainder of our work, all problems (analytical and numerical) are solved on a box-shaped domain referred to as the Carpinteri column which is shown in Fig. 1. The Carpinteri column is an ideal fractal body (having mathematically well defined Hausdorff dimensions in all three directions) that is embedded in a $3 \mathrm{~d}$ Euclidean space. It was considered by Carpinteri et al. [4] to model concrete columns which are essentially composite structures featuring an irregular (disordered) geometry. Concerning our application, the Carpinteri column is a meaningful illustration of a fractal solid body that exhibits the property of fractal anisotropy. The cross-section of the column, which is square in shape, constitutes a Sierpinski carpet. It is "fractaly" swept along the longitudinal direction in conjunction with a ternary Cantor set. Clearly, the Hausdorff dimension of this body along the longitudinal direction, $x_{3}$, is equivalent to that of the Cantor set. The figuring out of the Hausdorff dimensions for the in-plane directions $x_{1}$ and $x_{2}$ is not as evident as is the case with the longitudinal direction. Nevertheless, substantial knowledge and significant assumptions help conjecturing viable values for these dimensions. First, the axial and planar symmetries revealed in the Sierpiński carpet's geometry indisputably induce that $D_{1}=D_{2}$. 
This fractal set is built in a 2D Euclidean space and its Hausdorff dimension $\left(D_{\text {carpet }}=\frac{\ln 8}{\ln 3}\right)$ is derived within the $2 \mathrm{D}$ framework. Superficial knowledge in fractal geometry might lead to the erroneous assumption obtained by analogy with the continuous case where $D_{1}=\frac{1}{2} D_{\text {carpet }}$. The inapplicability of this simple supposition is briefly explained. The theory of products of fractal sets infers that if a fractal set (denoted as $G$ ) is generated from the Cartesian product of two primary fractal sets (denoted as $E$ and $F$ ), i.e. $G=E \times F$, their dimensions satisfy the following inequality (it becomes equality for continuous and Cantor sets),

$$
D_{G} \geq D_{E}+D_{F}
$$

See chap. 7 of [7] for more about this topic. Unluckily, the Sierpiński carpet is not formed as the product of two independent fractal sets. Thus Eq. (17) cannot even predict an upper limit for $D_{1}$. If we consider the horizontal slice (set formed by intersecting a horizontal line with the carpet) and attempt to obtain the dimension of that slice, we realize that it depends on the position where the line intercepts the carpet. For example, on side length corresponding to $x=\left\{0, \frac{1}{3}, \frac{2}{3}, 1\right\}, D_{\text {slice }}=1$ since no void is to occur at this position. On the other hand, if $x=\frac{1}{2}$, we obtain $D_{\text {slice }}=D_{\text {Cantor set }}$. Clearly, the dimension is not uniform along the edge, it is evaluated statistically in [16] and this is how $D_{1}$ was estimated for our problem. In conclusion, the utilized Hausdorff dimension along each direction is provided below.

$$
\begin{aligned}
& D_{1}=D_{2}=\frac{1}{3} \frac{\ln 18}{\ln 3} \\
& D_{3}=\frac{\ln 2}{\ln 3}
\end{aligned}
$$




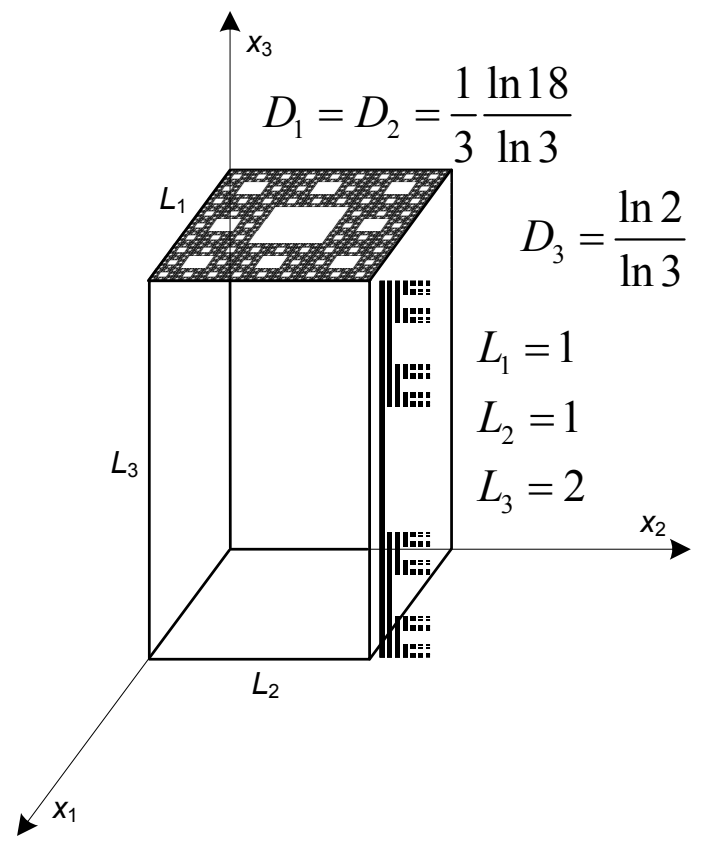

Figure 1: Layout of the Carpinteri column, the physical domain on which the dilatational wave propagation problem is solved. The Hausdorff dimensions are indicated for every direction.

The Hausdorff dimension for the entire column is given as

$$
D_{\text {Carp. col. }}=D_{\text {carpet }}+D_{3}=4 \frac{\ln 2}{\ln 3}
$$

This dimension is just a geometric property and plays no role in the mechanics under discussion. The Carpinteri column exhibits a planar fractal isotropy that renders the analysis along the $x_{1}$ and $x_{2}$ directions redundant especially since $L_{1}=L_{2}$.

\section{Modal analysis}

The propagation of dilatational waves is described in Eq. (16). We now present the analytical solution to this equation through modal analysis. The 
fulfilment of the modal analysis allows for the treatment of variety of boundary value problems (BVP), whereby we discuss three problems characterized by their distinct boundary conditions (BC). The solution to these problems will be later used in validating the computational tools constructed to simulate some complex problems which do not admit closed-form solutions.

Defining the dilatational wave speed to be

$$
\vartheta_{d}=\sqrt{\frac{\lambda+2 \mu}{\rho}}
$$

and expressing the displacement in its modal form

$$
u(x, t)=U(x) e^{j \omega t}
$$

The resulting modal equation, general for all directions, becomes

$$
-\left(\frac{U_{, x}}{c}\right)_{, x}=c k^{2} U
$$

or more explicitly,

$$
(L-x)^{2} U^{\prime \prime}(x)+(D-1)(L-x) U^{\prime}(x)+k^{2} D^{2}(L-x)^{2 D} U(x)=0
$$

where $k$ is the wavenumber

$$
k=\frac{\omega}{\vartheta_{d}}
$$

This modal equation admits the following general solutions (the derivation of the solution is explained in detail in the Appendix A)

$$
\begin{aligned}
& f_{1}(x, k)=\cos \left[k(L-x)^{D}\right] \\
& f_{2}(x, k)=\sin \left[k(L-x)^{D}\right]
\end{aligned}
$$


hence the modal function $U_{m}(x)$ for a given BVP can be expressed as the weighted sum of $f_{1}$ and $f_{2}$,

$$
U_{m}(x)=C_{1} f_{1}\left(x, k_{m}\right)+C_{2} f_{2}\left(x, k_{m}\right)
$$

where constants $C_{1}$ and $C_{2}$ are determined via application of the appropriate BC. In the case of homogeneous BC, the Sturm-Liouville problem presented in Eq. (22) admits the following modal orthonormality property

$$
\int_{0}^{L} U_{m}(x) U_{n}(x) c(x) \mathrm{d} x=\delta_{m n}
$$

This orthogonality is indeed verified in our discussed eigenvalue problems.

Having obtained the homogeneous solution to the general BVP, we now proceed to discuss three different eigenvalue type problems, each has its own setting of BC. For all these problems, the solutions to the modal functions and corresponding wavenumbers are evaluated.

\subsection{Dirichlet $B V P$}

In this section, we intend to solve the problem where homogeneous boundary conditions on $u$ are enforced on both sides of the domain. Thus, the modal function $U(x)$ must satisfy

$$
\left.U\right|_{x=0}=\left.0 \quad \& \quad U\right|_{x=L}=0
$$

Applying the boundary conditions of Eq. (28) into the general solution presented in Eq. (26), we obtain the non-trivial solution described as follows

$$
\begin{gathered}
U_{m}(x)=\sin \left[k_{m}(L-x)^{D}\right] \\
k_{m}=\frac{m \pi}{L^{D}} \quad m \in \mathbb{N}
\end{gathered}
$$




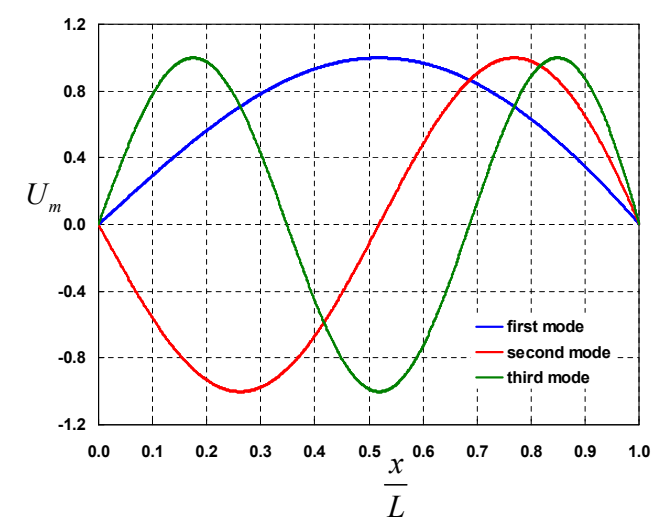

(a) $D=\frac{1}{3} \frac{\ln 18}{\ln 3}$

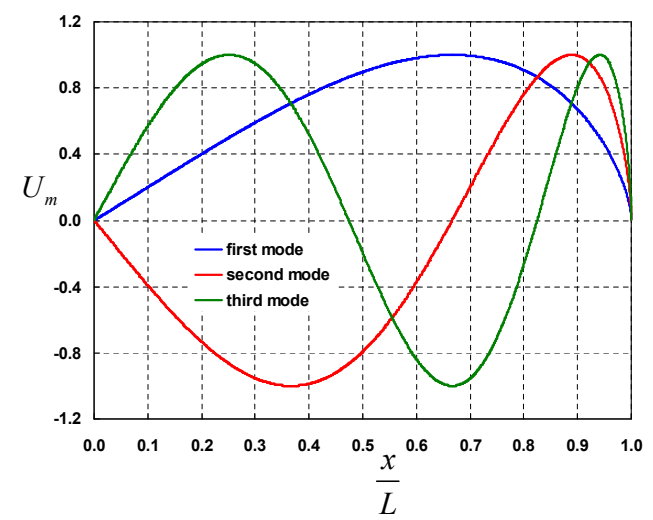

(b) $D=\frac{\ln 2}{\ln 3}$

Figure 2: The first three modal functions for the homogeneous Dirichlet BVP shown for Hausdorff dimensions corresponding to those of the Carpinteri column.

This general modal solution is valid for all three directions. It depends on $D$ and $L$ which are direction characteristics. By setting their appropriate values, the modal solution for a particular direction is obtained. The first three modal functions corresponding to the Carpinteri column are shown in Fig. 2. A thorough observation of the modal functions reveals that they are all infinitely steep at the right edge $(x=L)$. This is corroborated mathematically where we have

$$
\left.\frac{\mathrm{d} U_{m}}{\mathrm{~d} x}\right|_{x \approx L} \simeq(L-x)^{D-1}
$$

Per Eq. (30), the singularity of the first derivative of $U_{m}$ at the right edge intensifies for smaller $D$. This is clearly manifested in Fig. 2 where the plots become more "asymmetric" with respect to $x=0.5$ and squeezed near $x=L$ for higher modes and lower $D$. 


\subsection{First mixed $B V P$}

In this section, we consider the problem where a homogeneous Dirichlet $\mathrm{BC}$ is applied on the right side and a homogeneous Neumann BC on the left end. As a result, the following conditions

$$
\left.\frac{\mathrm{d} U}{\mathrm{~d} x}\right|_{x=0}=\left.0 \quad \& \quad U\right|_{x=L}=0
$$

must be satisfied in the general modal solution of Eq. (26). This eigenvalue problem admits the following solution

$$
\begin{aligned}
& U_{m}(x)=\sin \left[k_{m}(L-x)^{D}\right] \\
& k_{m}=\frac{(2 m+1) \pi}{2 L^{D}} \quad m \in \mathbb{N}
\end{aligned}
$$

The first three modal functions corresponding to the Carpinteri column are shown in Fig. 3. Similarly to the Dirichlet BVP, the modal functions are all infinitely steep at the right edge $(x=L)$. This is also corroborated mathematically where we have

$$
\left.\frac{\mathrm{d} U_{m}}{\mathrm{~d} x}\right|_{x \approx L} \simeq(L-x)^{D-1}
$$

Note that the steepness of the modal functions intensifies for higher modes

and lower $D$. In such a case, a coarse uniform mesh along $[0, L]$ may not sufficiently resolve the mode near the right edge, leading to poor numerical accuracy. This problem is remedied by applying a biased refinement in which the nodal concentration is higher near the right edge where steepness occurs. 


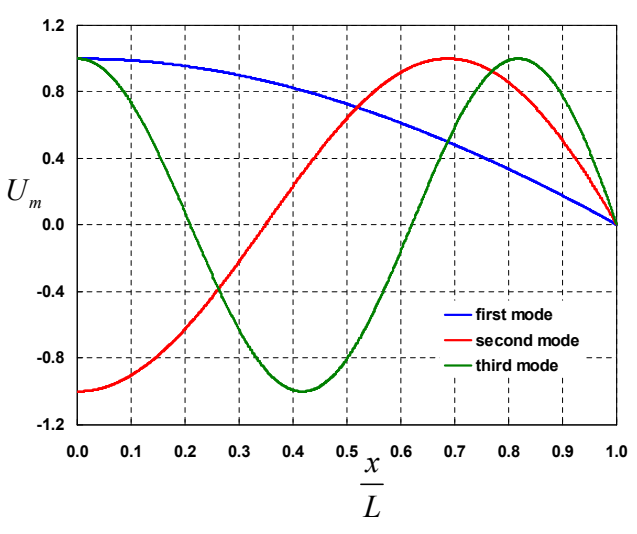

(a) $D=\frac{1}{3} \frac{\ln 18}{\ln 3}$

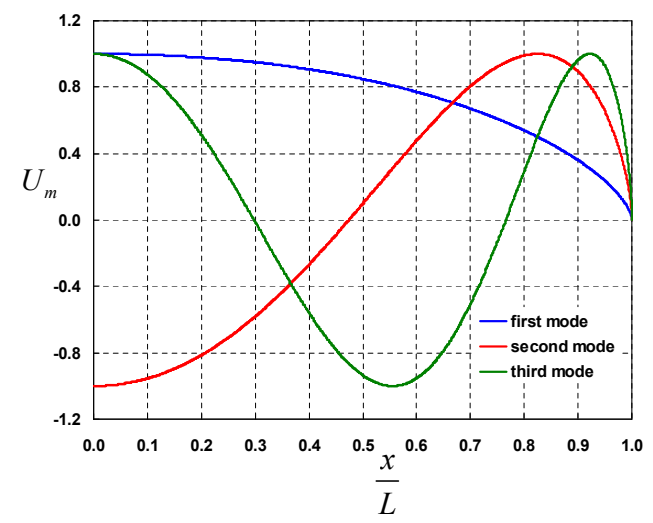

(b) $D=\frac{\ln 2}{\ln 3}$

Figure 3: The first three modal functions for the first mixed BVP shown for Hausdorff dimensions corresponding to those of the Carpinteri column.

\subsection{Second mixed BVP}

In this problem, we swap the locations of the $\mathrm{BC}$ applied in the first mixed BVP. In other words, we have

$$
\left.\frac{\mathrm{d} U}{\mathrm{~d} x}\right|_{x=L}=\left.0 \quad \& \quad U\right|_{x=0}=0
$$

At first glance, nothing about this problem should be different from its predecessor in terms of analysis and solution form. Nevertheless, the particularity of this problem lies in the fact that modal solutions exist only if $D>0.5$ as will be shown. For $D \leq 0.5$ the $\mathrm{BC}$ cannot be simultaneously applied and thus the eigenvalue problem has no solution. Applying the Neumann BC to the general solution of Eq. (26), we obtain

$$
\lim _{x \rightarrow L^{-}}\left[C_{1}(L-x)^{(D-1)} \sin \left(k(L-x)^{D}\right)-C_{2}(L-x)^{(D-1)} \cos \left(k(L-x)^{D}\right)\right]=0
$$




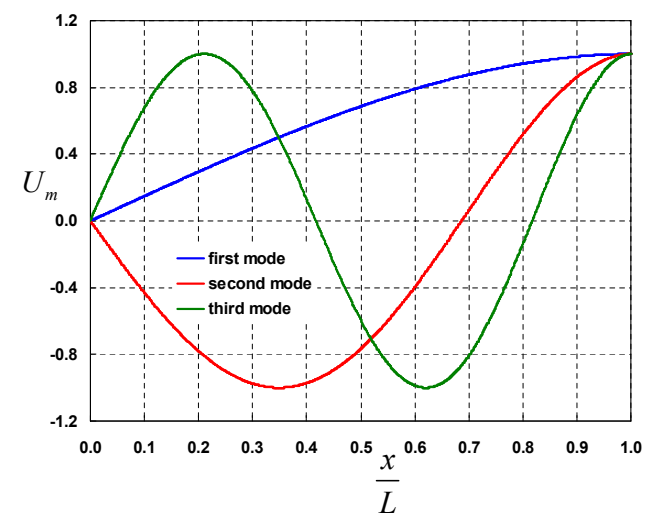

(a) $D=\frac{1}{3} \frac{\ln 18}{\ln 3}$

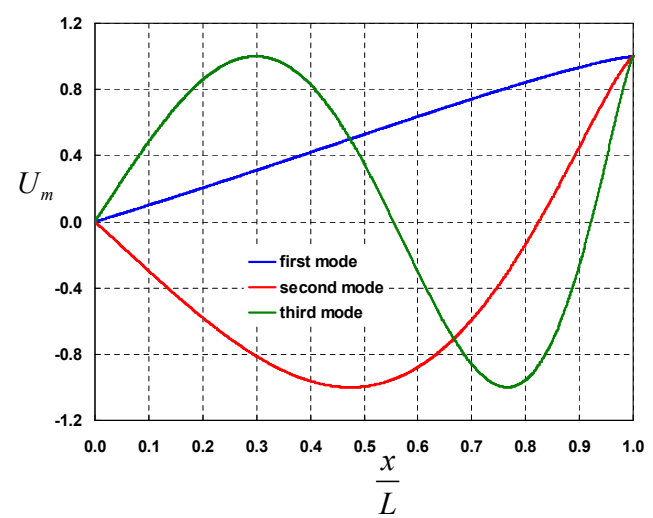

(b) $D=\frac{\ln 2}{\ln 3}$

Figure 4: The first three modal functions for the second mixed BVP shown for Hausdorff dimensions corresponding to those of the Carpinteri column.

the problem is now to solve for $C_{1}, C_{2}$, and $D$ for the limit to be satisfied. Clearly the second term of the equation diverges for any $D<1$, it thus must perish from the solution and this is achieved by setting $C_{2} \equiv 0$. The first term asymptotically behaves like $(L-x)^{(2 D-1)}$; it vanishes only if the power is positive, necessitating $D>0.5$. Concerning the wavenumber $k$, it is assigned via the application of the Dirichlet BC. In conclusion the modal solution is described as

$$
\begin{gathered}
U_{m}(x)=\cos \left[k_{m}(L-x)^{D}\right] \quad D>0.5 \\
k_{m}=\frac{(2 m+1) \pi}{2 L^{D}} \quad m \in \mathbb{N}
\end{gathered}
$$

The first three modal functions are shown in Fig. 4. Fortunately, the Hausdorff dimensions of our domain are all above 0.5 making the second mixed BVP simulation feasible. 


\section{Computational solutions}

The finite element method (FEM) is ubiquitously applied to simulate elastodynamic problems arising in mechanics of continuous materials $[1,10]$. In this work, we prove that FEM is robust in handling mechanics problems in fractal solids too. In the first subsection, we discuss the application of the FEM in solving the 1d problem resulting from decoupling of the governing equation. A transient analysis in addition to testing convergence in time and space are performed. Next, we simulate the $3 \mathrm{~d}$ problem in its full version, confirming the agreement between the $1 \mathrm{~d}$ and $3 \mathrm{~d}$ results.

\subsection{1d FEM problem}

As previously shown, the propagation of pure dilatational waves simplifies the general elastodynamic equation to three decoupled universal equations for all directions. Effectively, a large 3d problem can be reduced to three small 1d problems, each to be solved independently. This is a significant simplification from a computational perspective and it is worth exploiting as done below.

The reduced 1d equation (Eq. (16)) represents the strong form of the problem whose domain of interest is $\Omega=[0, L]$. This equation is first rewritten as

$$
\rho c \ddot{u}=(\lambda+2 \mu)\left(\frac{u_{, x}}{c}\right)_{, x}
$$

To obtain the weak form, we multiply the above equation by an admissible function $\hat{u}$ where $\hat{u} \in H^{1}(\Omega)$, the space of all test functions. Integrating over $\Omega$, we obtain

$$
\int_{\Omega} \rho c \ddot{u} \hat{u} \mathrm{~d} \Omega+\int_{\Omega}(\lambda+2 \mu) \frac{u_{, x} \hat{u}_{, x}}{c} \mathrm{~d} \Omega=0
$$


Partitioning the domain $\Omega$ into a countable union of subdomains (elements) $\Omega_{e}$, where $\bigcup_{e} \Omega_{e}=\Omega$, we obtain a discrete formulation based on two governing matrices, the inertia matrix $M$, and the stiffness matrix $K$. On the elemental level, these matrices are evaluated as

$$
\begin{gathered}
M_{i j}^{e}=\int_{\Omega_{e}} \rho c h_{i} h_{j} \mathrm{~d} x \\
K_{i j}^{e}=\int_{\Omega_{e}}(\lambda+2 \mu) \frac{h_{i, x} h_{j, x}}{c} \mathrm{~d} x
\end{gathered}
$$

As noticed, the governing matrices are symmetric; they can be evaluated exactly without performing any numerical integration (e.g. Gaussian quadrature rule). Once the assembly process is fulfilled, the discrete elastodynamic model is obtained, it is expressed as

$$
\mathbf{M} \cdot \ddot{\mathbf{U}}+\mathbf{K} \cdot \mathbf{U}=0
$$

The trapezoidal time stepping method is implemented to acquire the transient solution [10]. This time march scheme is implicit, unconditionally stable and second-order accurate. The numerical results for all three discussed problems are shown in Figures 5, 6, and 7. The Lamé constants, in addition to the density, are all set to one; the exciting frequencies are found accordingly. The solutions of both mixed BVP oscillate at the same frequency, and, indeed, their wavenumber forms are identical. All problems exhibit a remarkable agreement between the exact and numerical solutions.

The numerical solver is further validated in space and time to ensure convergence. We have analysed the decay power of $L_{2}$-norm of the global error with respect to element size and time step. For the spatial error, the convergence rate decreases as $D$ decreases most likely due to the skewness of the 


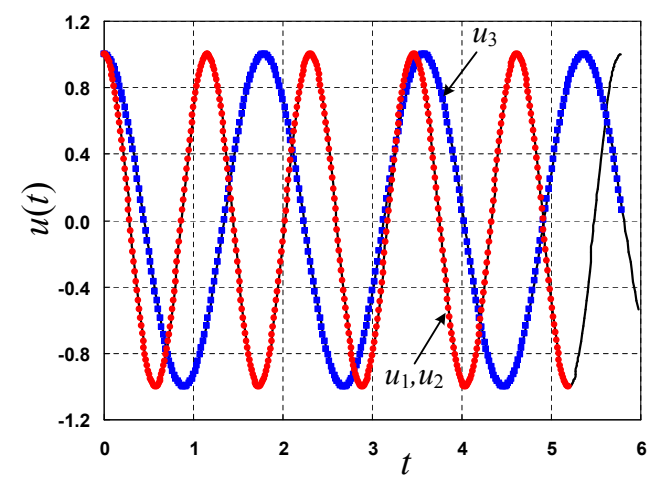

(a) first mode

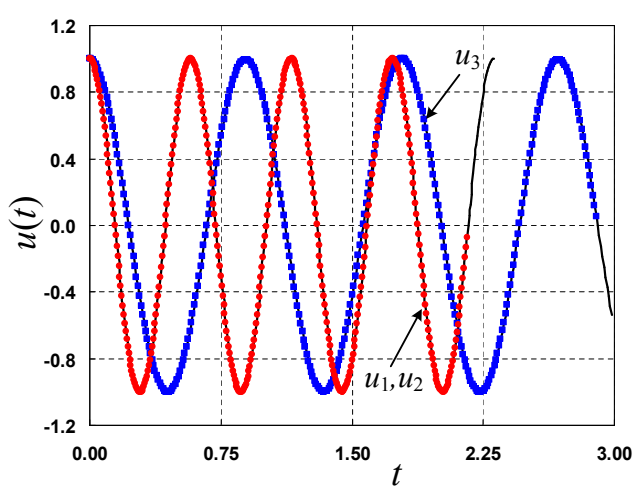

(b) second mode

Figure 5: Transient solution for the Dirichlet BVP. The first two modes are excited in which $\omega_{1,2}=5.44, \omega_{3}=3.51$ for the first mode and $\omega_{1,2}=10.88, \omega_{3}=7.03$ for the second mode. Frequencies are in rad/sec. Exact solutions are shown in solid line and numerical ones in coloured dots.

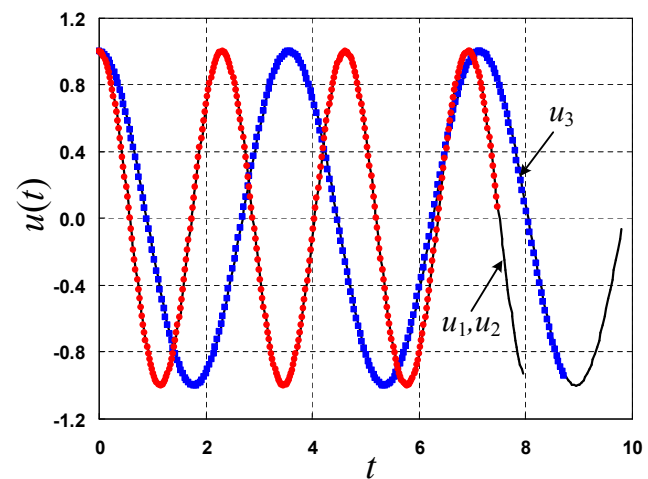

(a) first mode

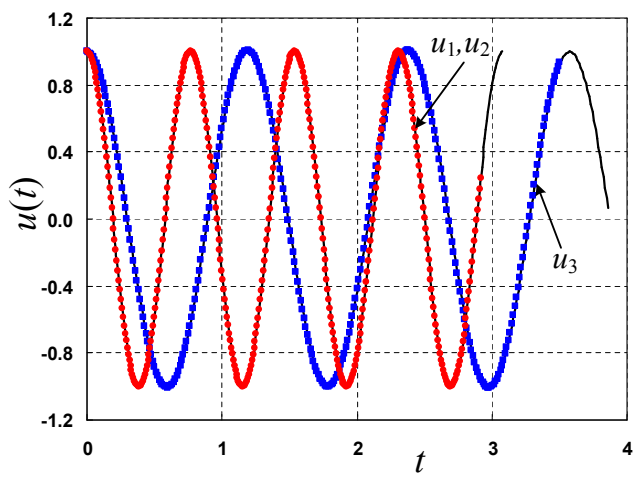

(b) second mode

Figure 6: The transient solution for the first mixed BVP. The first two modes are excited where $\omega_{1,2}=2.72, \omega_{3}=1.76$ for the first mode and $\omega_{1,2}=8.16, \omega_{3}=5.27$ for the second mode. Frequencies are in rad/sec. Exact solutions are shown in solid line and numerical ones in coloured dots. 


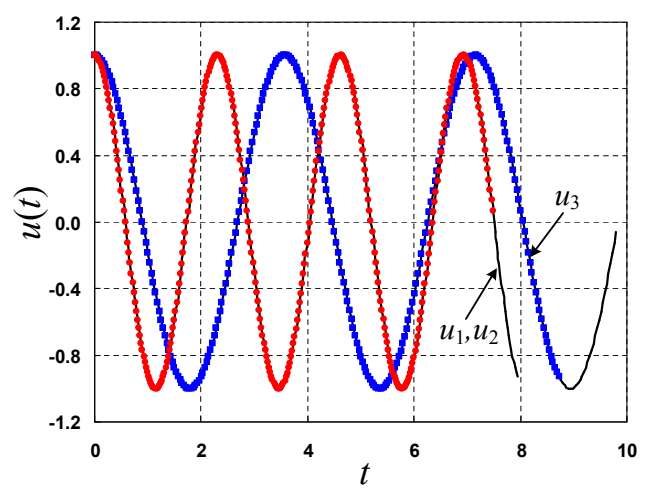

(a) first mode

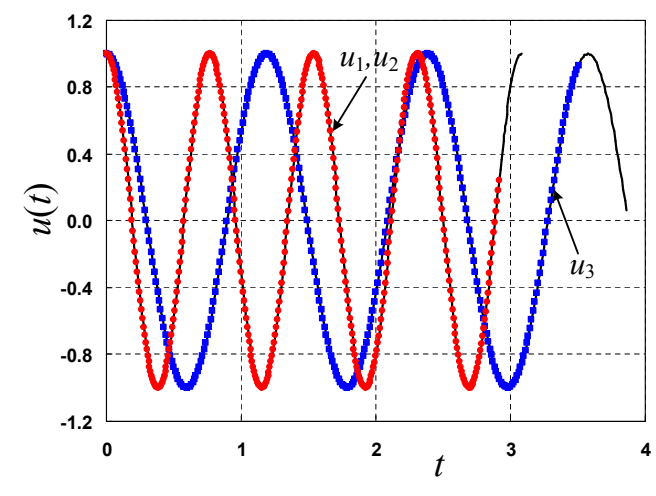

(b) second mode

Figure 7: The transient solution for the second mixed BVP. The first two modes are excited where $\omega_{1,2}=2.72, \omega_{3}=1.76$ for the first mode and $\omega_{1,2}=8.16, \omega_{3}=5.27$ for the second mode. Frequencies are in rad/sec. Exact solutions are shown in solid line and numerical ones in coloured dots.

mode shape for smaller $D$. The temporal error is nevertheless independent of $D$ and uniform for all the modes. The convergence plots are shown in Figures 8 and 9 .

\subsection{3d FDM analysis}

In this section, we ignore the computational advantage granted by the handling of dilatational waves which simplifies $3 \mathrm{~d}$ problems to only 1d. Effectively, we seek a numerical solution to Eq. (15) for a general problem setting. Even though waves of non-dilatational type can be analysed in this solver, numerical solutions for only dilatational wave problems are physically meaningful. Hence, the $3 \mathrm{~d}$ analysis adds no ingenuity to our computational work which, as shown in the previous section, can be adequately accomplished with the $1 \mathrm{~d}$ solver. Nevertheless, problems involving non-classical material models where general waves are permissible cannot be subject to 


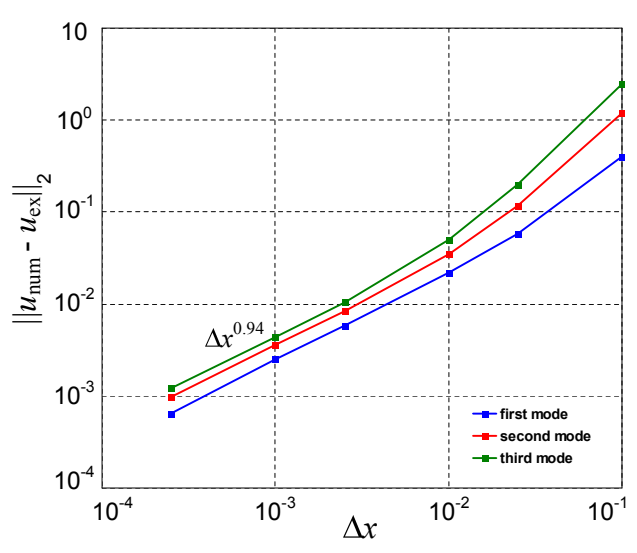

(a) $D=\frac{1}{3} \frac{\ln 18}{\ln 3}$

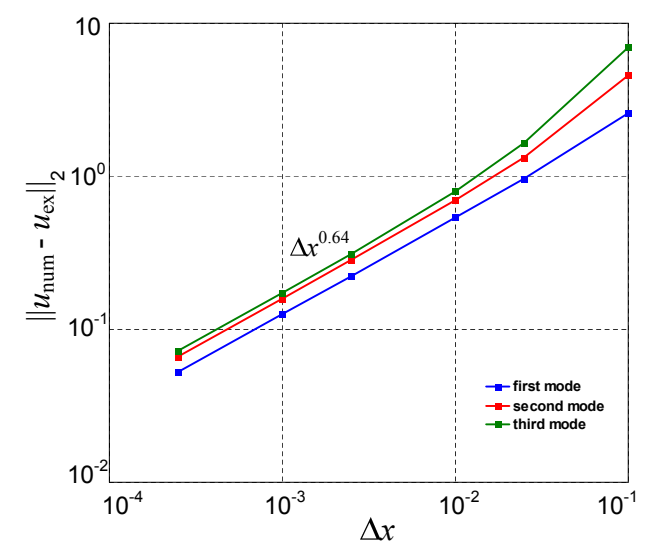

(b) $D=\frac{\ln 2}{\ln 3}$

Figure 8: Spatial convergence plots. The convergence analysis is based on the Dirichlet BVP. The effects of the Hausdorff dimension on convergence are clearly noticed.

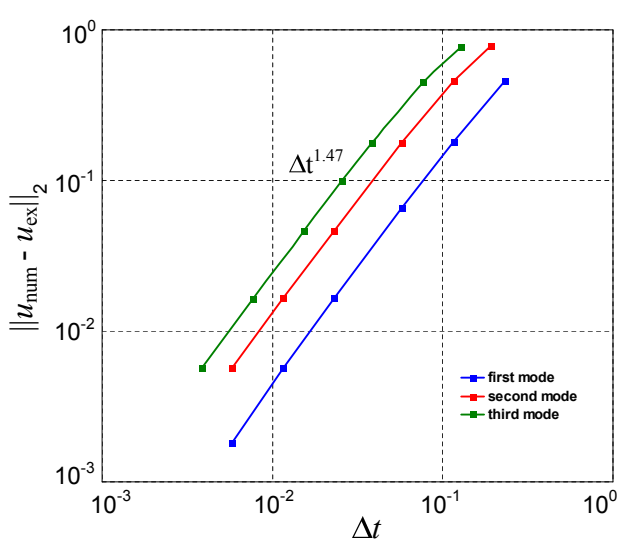

(a) $D=\frac{1}{3} \frac{\ln 18}{\ln 3}$

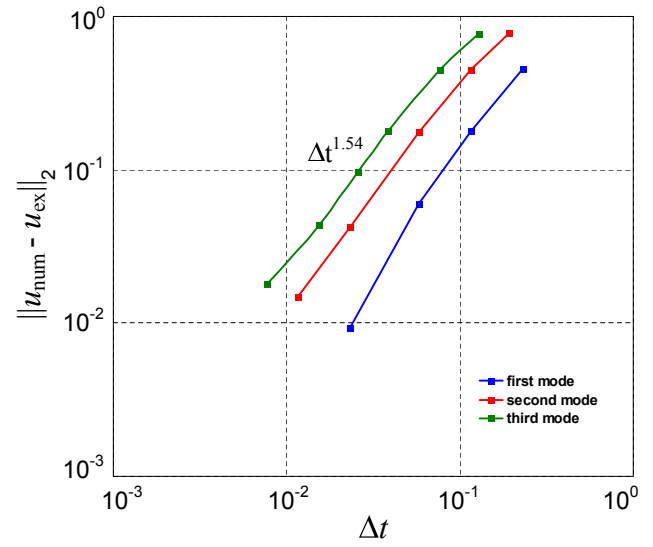

(b) $D=\frac{\ln 2}{\ln 3}$

Figure 9: Temporal convergence plots. The convergence analysis is based on the Dirichlet BVP. The Hausdorff dimension plays no role in altering the convergence power. 


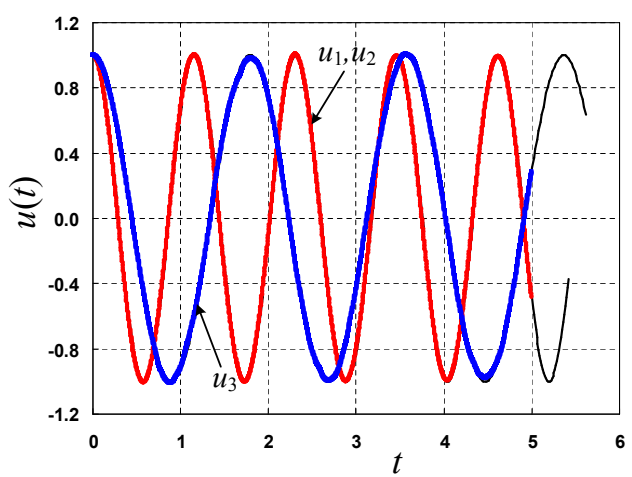

(a) first mode excitation

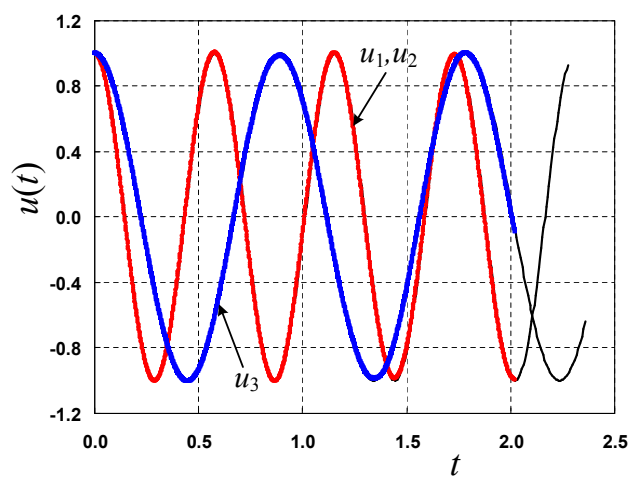

(b) second mode excitation

Figure 10: Results for 3d analysis. For the first mode we utilized $\Delta t=2.0 \times 10^{-3}$ and grid size $N_{x}=N_{y}=121, N_{z}=241$. For the second mode problem, the grid size is kept the same but $\Delta t=1.4 \times 10^{-3}$. Remarkable matching is observed between the exact (solid black) and the numerical (coloured dots) solution.

decoupling (as is the case here) and thus $3 \mathrm{~d}$ analysis becomes mandatory. Since we plan to consider these problems in the near future, we decided to briefly introduce this complex work in this paper which we can refer to in our upcoming research.

In the numerical framework, Eq. (15) can be most effectively handled in a finite difference discretization with explicit time march setting [6]. In brief, we adopted the $2^{\text {nd }}$-order accurate spatial discretization and the $1^{\text {st }}$-order accurate Euler's forward method to respectively approximate derivatives arising in elastic and inertia terms. Results for the first two modal excitations of the Dirichlet BVP are shown in Fig. 10. In addition, contour plots for the displacement field at different time steps are also shown in Figures 11 and 12 for the first modal excitation and in Figures 13 and 14 for the second modal excitation. 


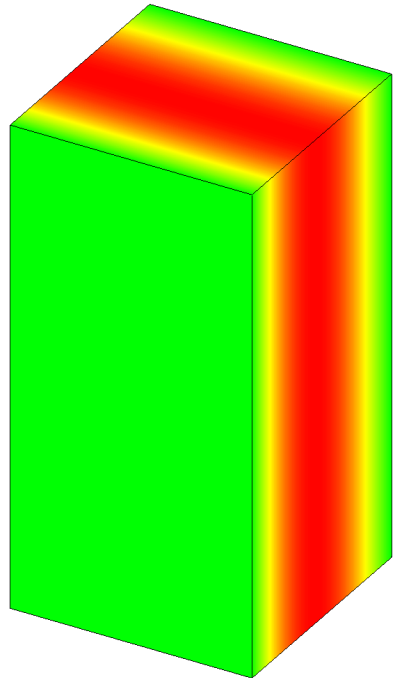

(a) $u_{1}$

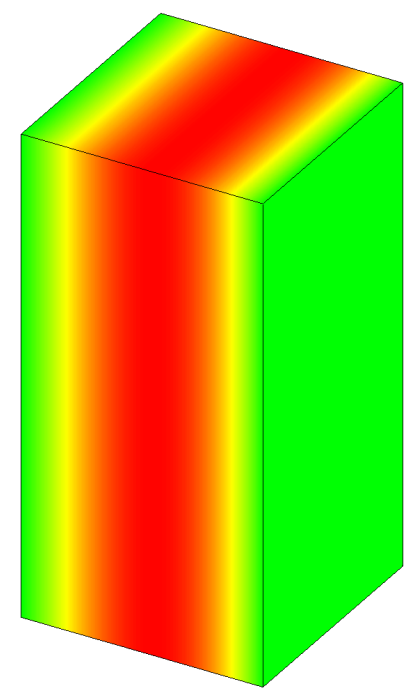

(b) $u_{2}$

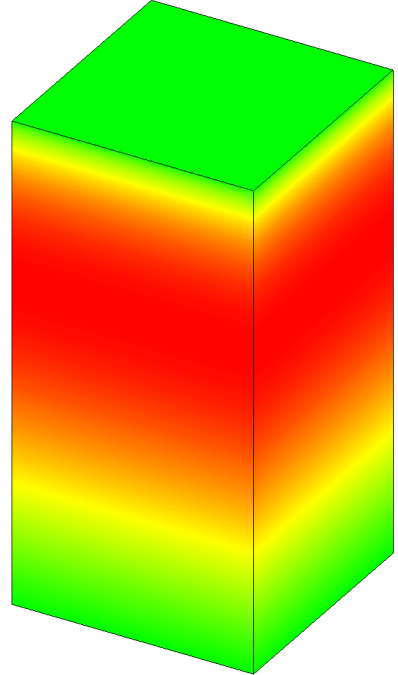

(c) $u_{3}$

Figure 11: Contour plots for the solution of the Dirichlet BVP in first mode excitation at $t=0 \mathrm{sec}$.

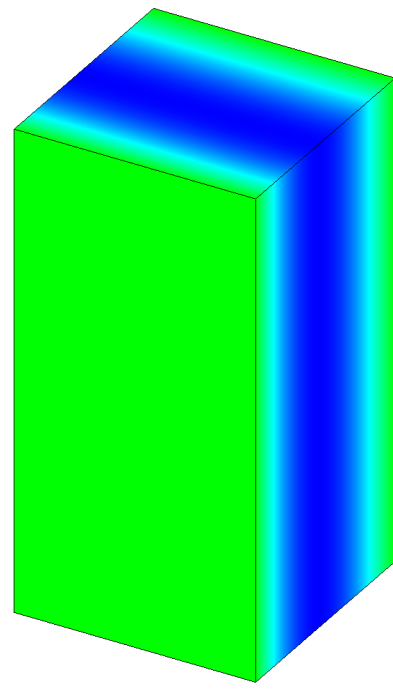

(a) $u_{1}$

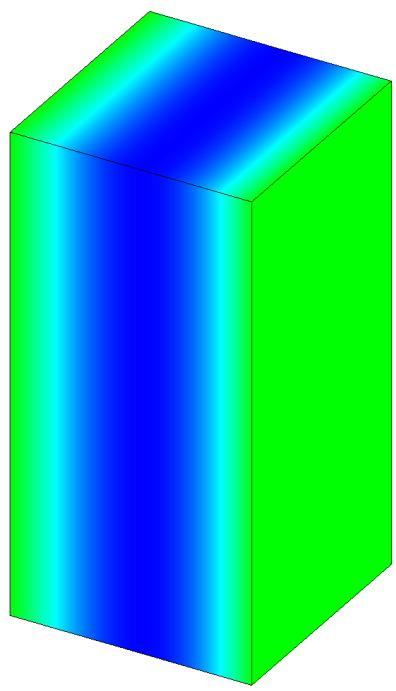

(b) $u_{2}$

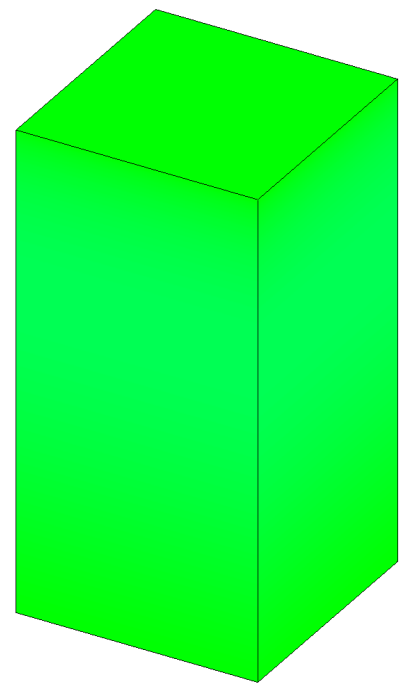

(c) $u_{3}$

Figure 12: Contour plots for the solution of the Dirichlet BVP in first mode excitation at $t=4 \mathrm{sec}$. 


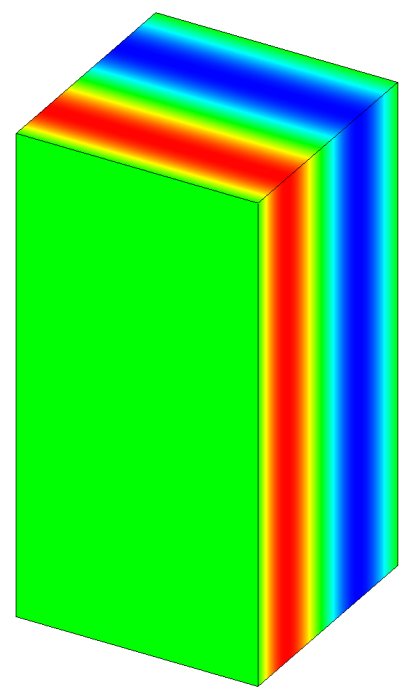

(a) $u_{1}$

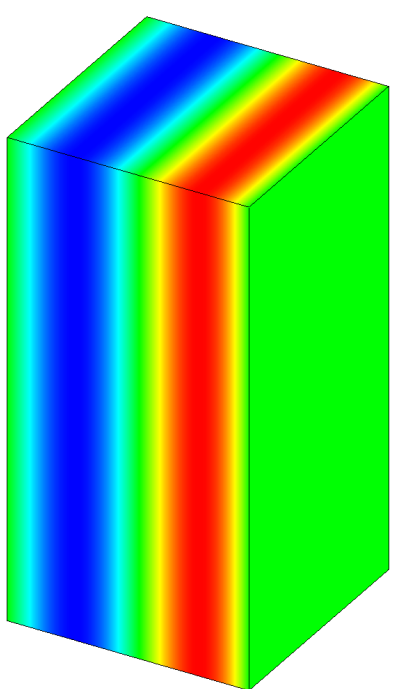

(b) $u_{2}$

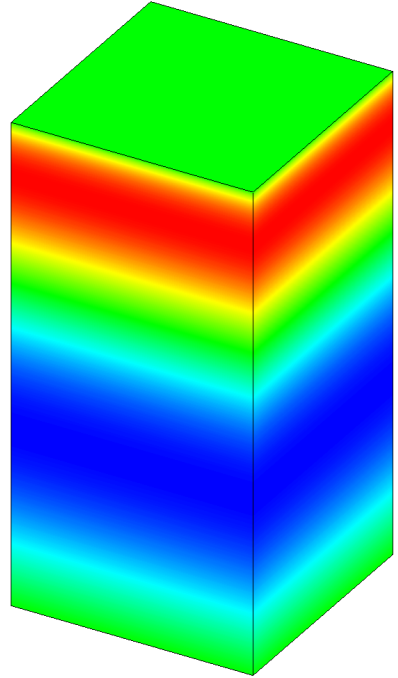

(c) $u_{3}$

Figure 13: Contour plots for the solution of the Dirichlet BVP in second mode excitation at $t=0 \mathrm{sec}$.

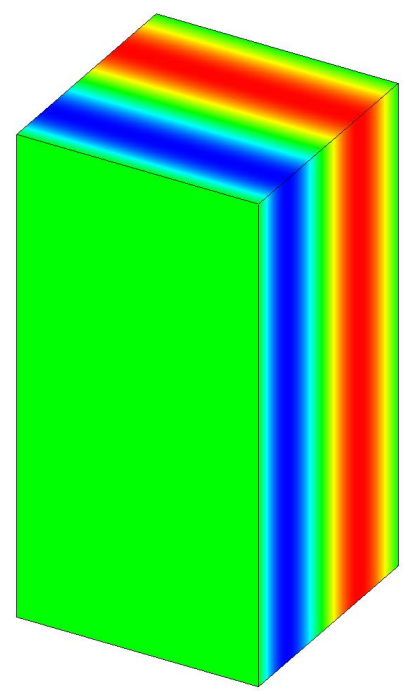

(a) $u_{1}$

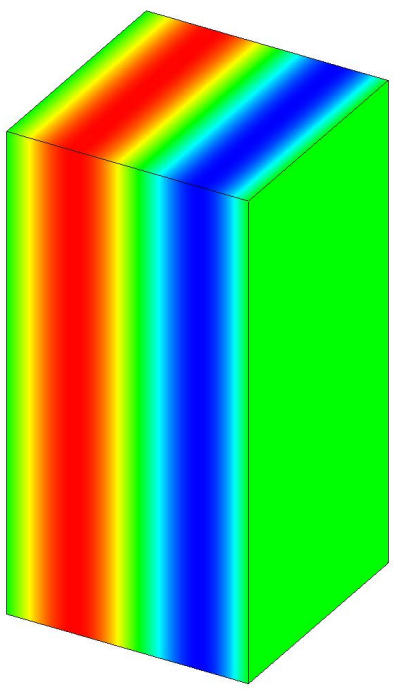

(b) $u_{2}$

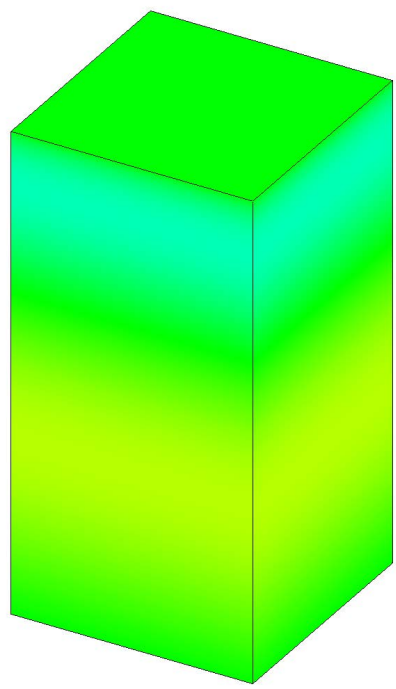

(c) $u_{3}$

Figure 14: Contour plots for the solution of the Dirichlet BVP in second mode excitation at $t=2 \mathrm{sec}$. 


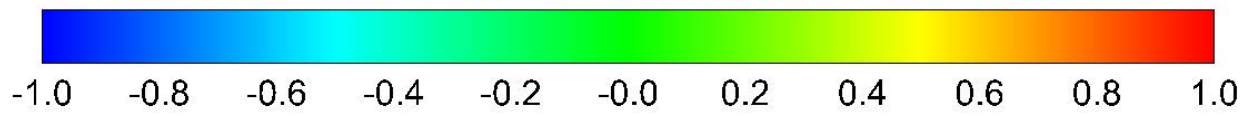

Figure 15: Legend scale for all contour plots shown in Figures 11, 12, 13, and 14.

\section{Conclusion}

This paper reports on the elastodynamics of solids with a generally anisotropic, product-like fractal structure, yet governed by an isotropic Hooke's law. This setting allows propagation of purely dilatational waves, and this is studied through dimensional regularization. The analytic work achieved through the eigenproblem analysis permits solving any BVP by modal superposition. We have also implemented a computational approach to analyse this wave problem, whereby a finite element method solver was developed and validated, and the transient response for a BVP based on modal excitation was evaluated. Finally, this work represents a valuable cornerstone in the currently active exploration of the mechanics of fractal materials. Its significance lies in the mathematical and computational study, which to a large extent, prompts the realization of the solution to the general elastodynamic problem where arbitrary waves are involved and non-classical constitutive models are incorporated [12].

\section{Acknowledgements}

This research was made possible by the support from Sandia-DTRA (grant HDTRA1-08-10-BRCWMD) and the NSF (grant CMMI-1030940). 


\section{Appendix A. Solution of the modal equation}

The modal ordinary differential equation (ODE) for the dilatational wave problem is given as

$$
(L-x)^{2} f^{\prime \prime}(x)+(D-1)(L-x) f^{\prime}(x)+k^{2} D^{2}(L-x)^{2 D} f(x)=0
$$

An obvious transformation to conceal the $L$ term is first applied, it is given as

$$
y=L-x
$$

the resulting ODE in terms of $y$ becomes

$$
y^{2} \frac{\mathrm{d}^{2} f}{\mathrm{~d} y^{2}}-(D-1) y \frac{\mathrm{d} f}{\mathrm{~d} y}+k^{2} D^{2} y^{2 D} f(y)=0
$$

The presence of the non-integer power in the last term deters a quick figuring out of a solution form. At this point, two approaches can be followed to determine the solution. The first, which is less likely to be noticed, requires the application of the following transformation

$$
z=y^{D}
$$

the resulting equation in $z$ becomes

$$
\frac{\mathrm{d}^{2} f}{\mathrm{~d} z^{2}}+k^{2} f(z)=0
$$

the clear solution to Eq. (A.5) is

$$
f(z)=C_{1} \cos (k z)+C_{2} \sin (k z)
$$

and consequently in terms of $x$, the solution is

$$
f(x)=C_{1} \cos \left[k(L-x)^{D}\right]+C_{2} \sin \left[k(L-x)^{D}\right]
$$


In case the second transformation cannot be easily noticed, there exists an alternative approach in which we seek a series solution to Eq. (A.3) of the form

$$
f(y)=\sum_{n=0}^{\infty} a_{n} y^{(n \alpha+\beta)}
$$

where $\alpha$ and $\beta$ are two independent constants, in general, non-integer. The attempt to have a series solution with pure integer powers fails because of the presence of a non-integer power in the ODE itself. The substitution of this series form into the ODE produces the following relations

$$
\begin{gathered}
\alpha=2 D \\
\beta= \begin{cases}0 & \text { or } \\
D\end{cases}
\end{gathered}
$$

For the first case, $\beta_{1}=0$, the recurrence relation for $a_{n}$ is given as

$$
a_{n}=-\frac{k^{2}}{2 n(2 n-1)} a_{n-1} \quad n \geq 1
$$

from which the general form of the $a_{n}$ term is deduced to be

$$
a_{n}=\frac{(-1)^{n} k^{2 n} \Gamma\left(\frac{1}{2}\right)}{4^{n} n ! \Gamma\left(n+\frac{1}{2}\right)} a_{0}
$$

As a result, the first homogeneous solution, $f_{1}$, in a series form becomes

$$
f_{1}(y)=a_{0} \Gamma\left(\frac{1}{2}\right) \sum_{n=0}^{\infty} \frac{(-1)^{n}}{n ! \Gamma\left(n+\frac{1}{2}\right)}\left(\frac{k y^{D}}{2}\right)^{2 n}
$$

Recognizing that the series form of the Bessel function of first kind and order $\nu, J_{\nu}(y)$, is given as [2]

$$
J_{\nu}(y)=\sum_{n=0}^{\infty} \frac{(-1)^{n}}{n ! \Gamma(n+1+\nu)}\left(\frac{y}{2}\right)^{2 n+\nu}
$$


The similarity of the forms in equations (A.12) and (A.13) permits the compaction of the first solution to $f_{1}(y) \simeq \sqrt{k y^{D}} J_{-0.5}\left(k y^{D}\right)$.

For the second case, $\beta_{2}=D$, the following recurrence relation for $a_{n}$ is obtained

$$
a_{n}=-\frac{k^{2}}{2 n(2 n+1)} a_{n-1} \quad n \geq 1
$$

which leads to a general form of $a_{n}$ given as

$$
a_{n}=\frac{(-1)^{n} k^{2 n} \Gamma\left(\frac{1}{2}\right)}{4^{n} n ! \Gamma\left(n+\frac{3}{2}\right)} a_{0}
$$

Consequently, the second homogeneous solution, $f_{2}$, admits the following series form

$$
f_{2}(y)=a_{0} \Gamma\left(\frac{1}{2}\right) y^{D} \sum_{n=0}^{\infty} \frac{(-1)^{n}}{n ! \Gamma\left(n+\frac{3}{2}\right)}\left(\frac{k y^{D}}{2}\right)^{2 n}
$$

Similarly to the first solution, the series form of $f_{2}$ is matched to that of Eq. (A.13), resulting in having $f_{2}(y) \simeq \sqrt{k y^{D}} J_{0.5}\left(k y^{D}\right)$. In conclusion, the general solution is

$$
f(y)=\sqrt{k y^{D}}\left[J_{-0.5}\left(k y^{D}\right)+J_{0.5}\left(k y^{D}\right)\right]
$$

We here note that the above solution is independently achieved in chap. 6 of [3] where a more general ODE is treated in term of Bessel functions.

In the following steps, we will bridge the general solutions from their Bessel function form to their equivalent harmonic form. For this proof, we rely on two important identities concerning Bessel functions. The first, is the 
Bessel integral given as

$$
J_{\nu}(y)=\frac{\int_{0}^{y}\left(y^{2}-\tau^{2}\right)^{\nu-0.5} \cos \tau \mathrm{d} \tau}{2^{\nu-1} \Gamma\left(\nu+\frac{1}{2}\right) \sqrt{\pi} y^{\nu}}
$$

and the second, is a recurrence relation defined as

$$
J_{\nu-1}(y)+J_{\nu+1}(y)=\frac{2 \nu}{y} J_{\nu}(y)
$$

From Eq. (A.18) we obtain $J_{0.5}(y)=\sqrt{\frac{2}{\pi}} \frac{\sin y}{\sqrt{y}}$ and $J_{1.5}(y)=\sqrt{\frac{2}{\pi}} \frac{\sin y-y \cos y}{y \sqrt{y}}$. Incorporating the above relations into Eq. (A.19), we obtain $J_{-0.5}(y)=$ $\sqrt{\frac{2}{\pi}} \frac{\cos y}{\sqrt{y}}$. Substituting the harmonic form of $J_{-0.5}$ and $J_{0.5}$ into Eq. (A.17), the solution form of Eq. (A.7) is reproduced.

\section{References}

[1] K.J. Bathe, Finite Element Procedures, Prentice Hall, NJ, 1982.

[2] C.M. Bender, S.A. Orszag, Advanced Mathematical Methods for Scientists and Engineers, Springer, 1999.

[3] F. Bowman, Introduction to Bessel Functions, Dover Publications, New York, 1958.

[4] A. Carpinteri, B. Chiaia, P. Cornetti, A disordered micro structure material model based on fractal geometry and fractional calculus, J. Appl. Math. Phys. (ZAMP) 84 (2004) 128-135.

[5] P.N. Demmie, M. Ostoja-Starzewski, Waves in fractal media, J. Elast. 104 (2011) 187-204. 
[6] D. Durran, Numerical Methods for Wave Equations in Geophysical Fluid Dynamics, Springer, New York, 1998.

[7] K. Falconer, Fractal Geometry: Mathematical Foundations and Applications, Wiley, England, 2003.

[8] K.F. Graff, Wave Motion in Elastic Solids, Dover Publications, New York, 1975.

[9] H. Hastings, G. Sugihara, Fractals: A User's Guide for the Natural Sciences, Oxford Science Publications, Oxford, 1993.

[10] T.J.R. Hughes, The Finite Element Method, Dover Publications, New York, 2000.

[11] H. Joumaa, M. Ostoja-Starzewski, On the wave propagation in isotropic fractal media, J. Appl. Math. Phys. (ZAMP) 62 (2011) 1117-1129.

[12] H. Joumaa, M. Ostoja-Starzewski, P.N. Demmie, Elastodynamics in micropolar fractal solids, Math. Mech. Sol. (2012). In press.

[13] G. Jumarie, Table of some basic fractional calculus formulae derived from a modified Riemann-Liouville derivative for non-differentiable functions, Appl. Math. Lett. 22 (2008) 378-385.

[14] J. Li, M. Ostoja-Starzewski, Fractal solids, product measures and fractional wave equations, Proc. R. Soc. Lond. A 465 (2009) 2521-2536.

[15] J. Li, M. Ostoja-Starzewski, Micropolar continuum mechanics of fractal media, Int. J. Eng. Sci. 49 (2011) 1302-1310. 
[16] A. Manning, K. Simon, Dimension of slices through the Sierpinski carpet, Transactions of the American Mathematical Society (2011). To appear.

[17] I.S. Sokolnikoff, R.M. Redheffer, Mathematics of Physics and Modern Engineering, Mc-Graw Hill Book Company, New York, 1958.

[18] V.E. Tarasov, Fractional hydrodynamic equations for fractal media, Ann. Phys. 318 (2005) 286-307. 